\title{
Control of Cascaded H-Bridge Multilevel Inverter Based on Optimum Regulation of Switching Angles, with FPGA Implementation
}

\author{
Hatef Firouzkouhi
}

\begin{abstract}
A new concept in control of cascaded H-Bridge multi-level inverters is proposed in this paper. According to this concept, switching angles are considered to be independent from the output fundamental voltage. A polynomial term is presented to show the relation between switching angles and DC voltages. Based on this concept, Total Harmonic Distortion (THD) calculations are updated and proved to be independent from the fundamental voltage. Thus, once calculated for minimum THD, the switching pattern can be used for any required level of output voltage. To examine the effectiveness of the proposed method, it is applied in control of an eleven level inverter. The simulation results are demonstrated and verified through experiments with a setup controlled by Xilinx SPARTAN3 family FPGA (XC3S400-PQG208).
\end{abstract}

Index Terms-Cascaded H-Bridge multilevel inverter, Genetic Algorithm (GA), optimization, Total Harmonic Distortion (THD), FPGA.

\section{INTRODUCTION}

Multi-level inverters have come a long way since the first time proposed for High Voltage DC applications. In last few years they have been widely used in electric motor drives [1], renewable energies integration into power systems [2], [3] charging infrastructures for battery system [4], and flexible AC transmission (FACTS) systems [5], [6]. The authors in [5] proposed a fault detection method that is capable of detecting low fault current due to the inverters limitations. Low voltage ride through (LVRT), harmonic suppression, frequency stability, and fault ride through (FRT) are among the advantages of application of multi-level inverters into power system. In [7], FRT capability of the generators are analyzed in a hybrid transmission line including an overhead line and an underground cable and a novel data-mining based method is proposed for fault location which is very fast and accurate. Several multilevel inverter topologies have been introduced over the past decades [8], [9]. Due to its simplicity, modularity, and module level maximum power point tracking, cascaded H-bridge multilevel inverter topology has become an attractive configuration over the past years. This topology is seen as the most suitable topology for the integration of the renewable energies to power system. Existence of separate DC sources in cascaded multilevel inverter makes it possible to be directly fed by PV panels, wind turbine and fuel cells. Moreover, energy storage systems such as batteries can also be incorporated into hybrid systems using this topology. This has been accepted as a functional way to compensate the uncertainties of solar and wind energy generation. An effective method presented in research [10] shows inverter interfaced distributed energy sources can successfully mitigate the frequency excursions due to uncertainties of solar and wind generation.

To fulfill the goals associated with applications of cascaded H-bridge multilevel inverters, design and implementation of their switching technique plays the key role. Reducing harmonics is one of the most important requirements associated with control of cascaded H-bridge multilevel inverters. Thus, study on switching patterns for this topology have attracted the attention of researchers [11]. By properly controlling the switching pattern, Total Harmonic Distortion (THD) can effectively be reduced. Several well-known switching techniques have been introduced over the past two decades to control the output voltage of the inverter while minimizing THD of the output waveform. These techniques include sinusoidal pulse width modulation (SPWM) [12], carrier pulse width modulation [13], space vector pulse width modulation (SVPWM) [14], and selected harmonic elimination pulse width modulation (SHEPWM) [15].

In this paper, a novel method is proposed to determine the switching pattern for a typical cascaded multilevel inverter with unequal DC sources. It has been tried to find the optimum relevance between DC sources and switching angles which leads to minimum value of THD, while the fundamental content of output voltage can be flexibly controlled. The relation between switching angles and DC voltages is estimated through a polynomial. The fundamental voltage and other harmonic contents can be controlled through the optimum switching angles. The proposed method is straightforward and reduces the computational burden of calculating the control parameters of the system. The optimization results are found through Genetic Algorithm optimization method, and effectiveness of the proposed method is verified through an experimental set up and using a Xilinx SPARTAN3 FPGA.

\section{PROPOSED POLYNOMIAL ESTIMATION METHOD}

The configuration of a typical cascaded H-bridge multilevel inverter is shown in Fig. 1. Due to series connection of the H-Bridges, the output voltage of the cascaded $\mathrm{H}$-bridge multilevel inverter is given by

$$
V_{o}=V_{o 1}+V_{o 2}+\ldots+V_{o k}
$$

Hatef Firouzkouhi of Master of Science student at University of Nevada, Reno. ( email: h.f.kouhi@gmail.com ) 


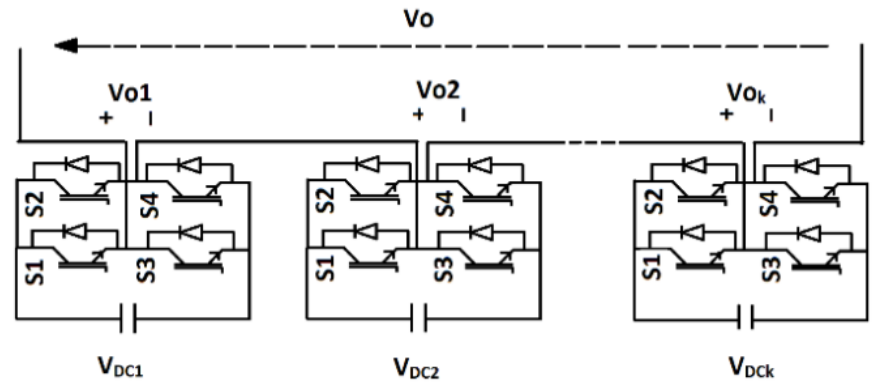

Fig 1: Cascaded multi-level inverter with $\mathrm{k}$ H_Bridges

Different switching patterns for power electronic switches of $S_{1}$ to $S_{4}$ generates $+V_{D C},-V_{D C}$, and zero at the output of individual H-Bridges. A typical cycle waveform with switching angles and voltage levels is shown in Fig. 2.

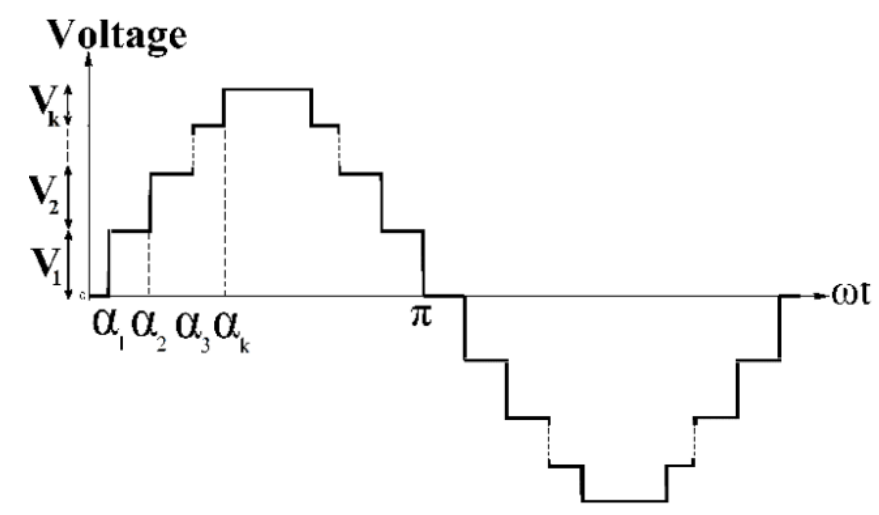

Fig 2: Stair case output voltage of a cascaded inverter with $\mathrm{k}$ H_Bridges

Fourier Transform is commonly used as a powerful tool for frequency analyzing for stepwise waveforms. The Fourier series of the symmetric waveform in Fig. 2 is given as

$$
V(\omega t)=\sum_{n=1,3,5, \ldots}^{\infty} V_{n} \sin (n \omega t)
$$

Where $V_{n}$ is the amplitude of $\mathrm{n}^{\text {th }}$ voltage harmonics as

$V_{n}= \begin{cases}\frac{4}{n \pi} \sum_{k} V_{D C k} \cos \left(n \alpha_{k}\right) & \text { For odd } \mathrm{n} \\ 0 & \text { For even } \mathrm{n}\end{cases}$

where $\mathrm{k}$ is the number of $\mathrm{H}$-bridges and $\mathrm{V}_{\mathrm{DCk}}$ is the voltage of the $\mathrm{k}^{\text {th }} \mathrm{DC}$ source connected that $\mathrm{H}$-Bridge. Even harmonic orders do not exist in symmetric waveforms, and THD is generally formulated as follow

$T H D=\frac{\sqrt{\sum_{n=3}^{\infty} V_{n}^{2}}}{V_{1}}$

Where $\mathrm{V}_{1}$ is the first harmonic of the output voltage called fundamental component in this paper. However, taking all harmonics into account is a big problem. Thus, low-order harmonics until a specified harmonic are considered so that an approximate answer is yielded. In this paper THD is calculated until $31^{\text {th }}$ order.

In this paper, it has been tried to independently control switching angles from the output fundamental voltage. Therefore, once obtained, the switching pattern is expandable for any required output voltage. In this paper a polynomial form is considered for the relation between switching angles and their corresponding H-Bridge DC voltage. In this case, the DC voltage sources are set as

$V_{D C k}=C * V_{1} * b_{k}$

$\mathrm{b}_{\mathrm{k}}$ is the polynomial defined as

$b_{k}=\sum_{i} A_{i} * \alpha_{k}^{i}$

By substituting (5) in (3) for $n=1$, we will have

$V_{1}=\frac{4}{\pi} \sum_{k} C V_{1} * \mathrm{~b}_{\mathrm{k}} * \cos \left(\alpha_{k}\right)$

Where $\mathrm{C}$ is a constant which is found as

$C=\frac{\pi}{4} \frac{1}{\sum_{k} \mathrm{~b}_{\mathrm{k}} * \cos \left(\alpha_{k}\right)}$

By substituting $\mathrm{C}$ in the (3), $\mathrm{n}^{\text {th }}$ voltage harmonic is obtained as follows

$V_{n}=\frac{4}{n \pi} \sum_{k} C * V_{1} * b_{k} * \cos \left(n \alpha_{k}\right)$

By substituting (9) in (4), THD is calculated by

$T H D=\frac{4 C}{\pi} \sqrt{\sum_{n=5,7,11, . .}^{31}\left(\sum_{i=1}^{k} \frac{b_{k} * \cos \left(n \alpha_{k}\right)}{n}\right)^{2}}$

This equation proves that THD can be calculated independent from the fundamental voltage.

\section{RESULTS AND DISCUSSION}

Genetic Algorithm (GA) is used as the optimization algorithm in this paper. GA is a stochastic global search tool which has been widely used in solving complicated optimization problems. Pattern recognition and optimization algorithms can be addressed through diverse computational, and combinatorial models such as cross efficiency in Data Envelopment Analysis (DEA) [16], and decision tree and association rules in data mining [17]. As a heuristic optimization method, GA does not require any derivative information. Thus, it reduces the computational burden of solving the problem.

In this paper, GA is used to define the optimal switching angles $\alpha_{k}$, and polynomial constants $A_{i} .100$ trial and error runs are performed using MATLAB, and the best result is introduced as the final optimum solution. The Objective function is considered as (10) to minimize the output THD. The optimum solution must satisfy the following constraint for switching angles.

$$
\alpha_{1}<\alpha_{2}<\ldots<\alpha_{k}<\frac{\pi}{2}
$$

To examine the effectiveness of the proposed estimation method, it has been applied in switching of an eleven-level single phase cascaded $\mathrm{H}$-Bridge inverter. The optimization results are shown in Table. I. Fig. 3 shows convergence flow of the optimization using GA. Elite count and crossover fraction for this simulation were set to 1 and 0.9 , respectively. 
The population size, were also set to 2000 .

These results can be applied for the control of the inverter for any desired fundamental voltage, and indicate that the switching angles are independent from the fundamental voltage values. DC voltages can be calculated using pre- defined switching angles for any desired fundamental output voltage. Fig. 4 shows calculated DC voltages for the whole possible range of fundamental voltage from zero to 1 P.U, using the results of Table. I.

Table I: Optimization results for eleven-level inverter

\begin{tabular}{|c|c|c|c|c|c|c|c|c|c|c|c|c|c|c|c|}
\hline THD\% & $\mathbf{A}_{1}$ & $\mathbf{A}_{2}$ & $\mathbf{A}_{3}$ & $\mathbf{A}_{4}$ & $\mathbf{A}_{5}$ & $\mathbf{A}_{6}$ & $\mathbf{A}_{7}$ & $\mathbf{A}_{8}$ & $\mathbf{A}_{9}$ & $\mathbf{A}_{10}$ & $\begin{array}{c}\alpha_{1} \\
\text { (Degrees) }\end{array}$ & $\begin{array}{c}\alpha_{2} \\
\text { (Degrees) }\end{array}$ & $\begin{array}{c}\alpha_{3} \\
\text { (Degrees) }\end{array}$ & $\begin{array}{c}\alpha_{4} \\
\text { (Degrees) }\end{array}$ & $\begin{array}{c}\alpha_{5} \\
\text { (Degrees) }\end{array}$ \\
\hline 4.95 & 0.03 & -0.85 & 1.11 & -0.20 & 0.81 & 0.99 & 1.95 & 0.73 & -2.21 & -2.05 & 5 & 15 & 26 & 38 & 58 \\
\hline
\end{tabular}

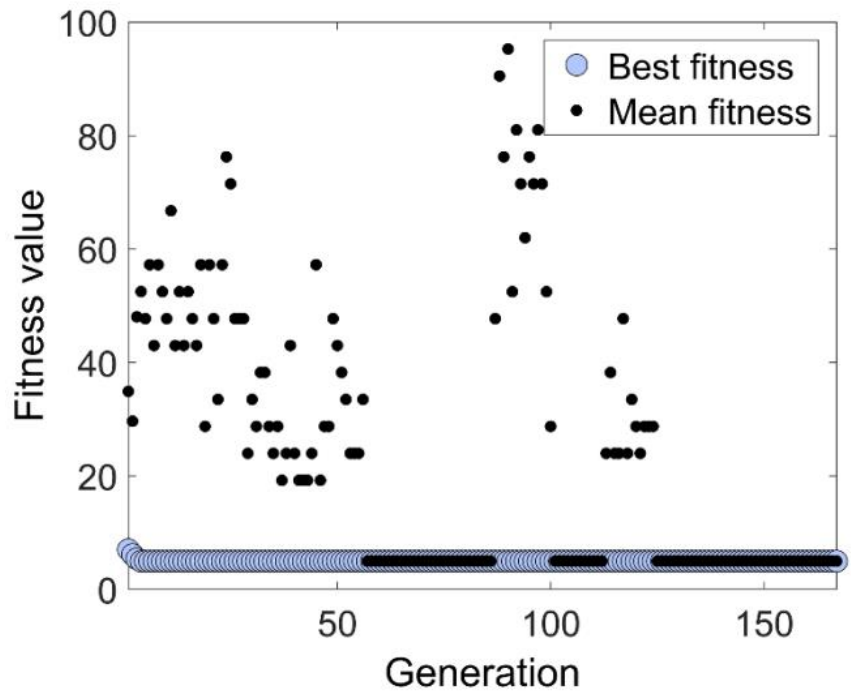

Fig 3: Genetic Algorithm convergence flow

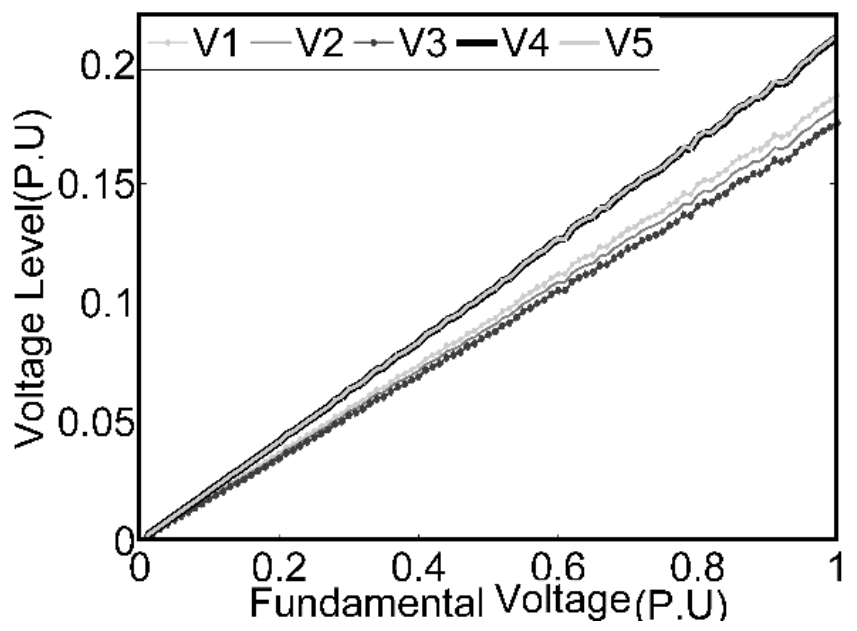

Fig 4: DC voltage sources against $\mathrm{V}_{1}$

To better show the effectiveness of the proposed method, three sample values for fundamental voltage is considered to represent low, medium and high levels of output voltage. DC voltages are calculated accordingly as shown in Table. II. Analysis of the results of this table reveals the ratio of voltage levels with respect to the fundamental voltage is constant, which is shown in Table. III.
Table II: DC voltage values versus different output voltage levels

\begin{tabular}{|c|c|c|c|c|c|}
\hline & $\mathbf{V}_{\text {DC1 }}$ & $\mathbf{V}_{\text {DC2 }}$ & $\mathbf{V}_{\text {DC3 }}$ & $\mathbf{V}_{\text {DC4 }}$ & $\mathbf{V}_{\text {DC5 }}$ \\
\hline 1 P.U & 0.19 & 0.18 & 0.17 & 0.21 & 0.22 \\
\hline 0.5P.U & 0.09 & 0.09 & 0.085 & 0.10 & 0.11 \\
\hline 0.1P.U & 0.019 & 0.018 & 0.017 & 0.021 & 0.022 \\
\hline
\end{tabular}

Table III: Voltage level ratios

\begin{tabular}{|c|c|c|c|c|}
\hline $\mathbf{V}_{\mathbf{D C} /} / \mathbf{V}_{\mathbf{1}}$ & $\mathbf{V}_{\mathbf{D C} 2 /} \mathbf{V}_{\mathbf{1}}$ & $\mathbf{V}_{\mathbf{D C} 3 /} \mathbf{V}_{\mathbf{1}}$ & $\mathbf{V}_{\mathbf{D C} 4 /} \mathbf{V}_{\mathbf{1}}$ & $\mathbf{V}_{\mathbf{D C} 5 /} \mathbf{V}_{\mathbf{1}}$ \\
\hline 0.19 & 0.18 & 0.17 & 0.21 & 0.22 \\
\hline
\end{tabular}

The Obtained harmonic spectra using switching angles and DC voltages from Tables. I-II are shown in Fig. 5. Analysis of the results show that the THD is kept at the minimum value for all three levels of output. Moreover, the magnitudes of individual harmonic orders are kept proportional to the fundamental voltage, and regardless of the fundamental voltage's value, a unified specified harmonic condition is preserved. This results are analysed in Table. IV.

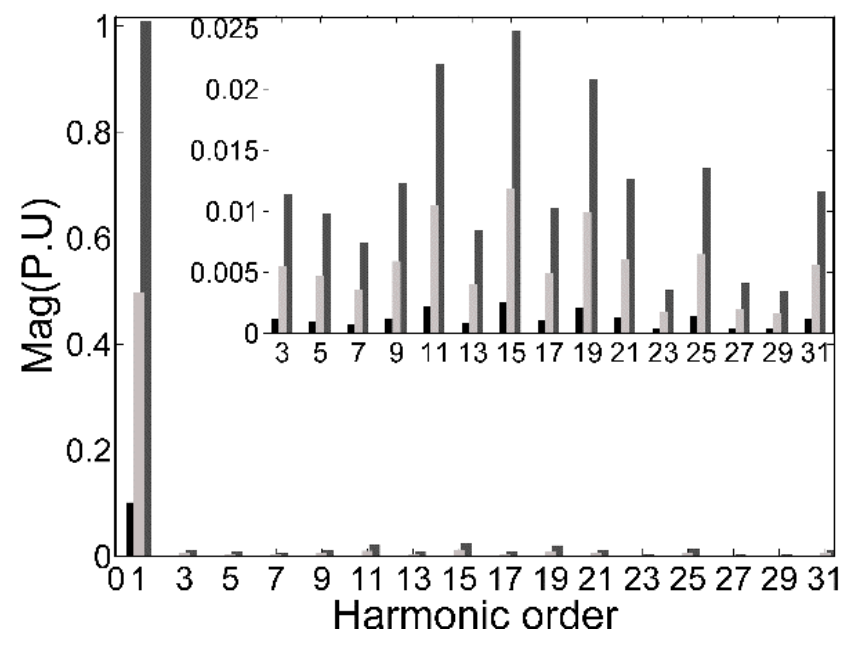

Fig 5: Harmonic spectra for three levels of fundamental voltage 
Table IV: Voltage harmonic ratios

\begin{tabular}{|l|l|l|l|l|l|l|l|l|l|l|l|l|l|l}
\hline $\mathbf{V}_{\mathbf{3}} / \mathbf{V}_{\mathbf{1}}$ & $\mathbf{V}_{\mathbf{5}} / \mathbf{V}_{\mathbf{1}}$ & $\mathbf{V}_{\mathbf{7}} / \mathbf{V}_{\mathbf{1}}$ & $\mathbf{V}_{\mathbf{9}} / \mathbf{V}_{\mathbf{1}}$ & $\mathbf{V}_{\mathbf{1 1}} / \mathbf{V}_{\mathbf{1}}$ & $\mathbf{V}_{\mathbf{1 3}} / \mathbf{V}_{\mathbf{1}}$ & $\mathbf{V}_{\mathbf{1 5}} / \mathbf{V}_{\mathbf{1}}$ & $\mathbf{V}_{\mathbf{1 7}} / \mathbf{V}_{\mathbf{1}}$ & $\mathbf{V}_{\mathbf{1 9}} / \mathbf{V}_{\mathbf{1}}$ & $\mathbf{V}_{\mathbf{2 1}} / \mathbf{V}_{\mathbf{1}}$ & $\mathbf{V}_{\mathbf{2 3}} / \mathbf{V}_{\mathbf{1}}$ & $\mathbf{V}_{\mathbf{2 5}} / \mathbf{V}_{\mathbf{1}}$ & $\mathbf{V}_{\mathbf{2 7}} / \mathbf{V}_{\mathbf{1}}$ & $\mathbf{V}_{\mathbf{2 9}} / \mathbf{V}_{\mathbf{1}}$ & $\mathbf{V}_{\mathbf{3 1}} / \mathbf{V}_{\mathbf{1}}$ \\
\hline $1.13 \%$ & $0.97 \%$ & $0.74 \%$ & $1.22 \%$ & $2.18 \%$ & $0.83 \%$ & $2.45 \%$ & $1.02 \%$ & $2.06 \%$ & $1.25 \%$ & $0.36 \%$ & $1.34 \%$ & $0.41 \%$ & $0.34 \%$ & $1.15 \%$ \\
\hline
\end{tabular}

\section{EXPERIMENTAL RESULTS}

Fig. 6 shows experimental setup for a single phase multilevel inverter. It consists of five $\mathrm{H}$-bridges which are connected in a series form. A Xilinx SPARTAN3 FPGA (XC3S400-PQG208) is used in the setup for the control of the gate drivers of power electronic switches. FPGA has high accuracy and speed in implementation of switching patterns. This FPGA is programmed using the open-source software 'Xilinx ISE 12.3'. After protection and isolation, the switching signals are transferred to gate drivers. FPGA, drivers and isolation circuits are placed on the controller board. More details regarding the experimental set up can be found in [18]. Switching angles are obtained according to Table. I. Figs. 7-9 show the experimental waveforms versus simulated waveforms for fundamental output voltage of 0.1 P.U, 0.5P.U and 1P.U. The base voltage used for calculation of these values is set to 10 volts. As can be inferred from the results, the measured waveforms verify the simulation results.

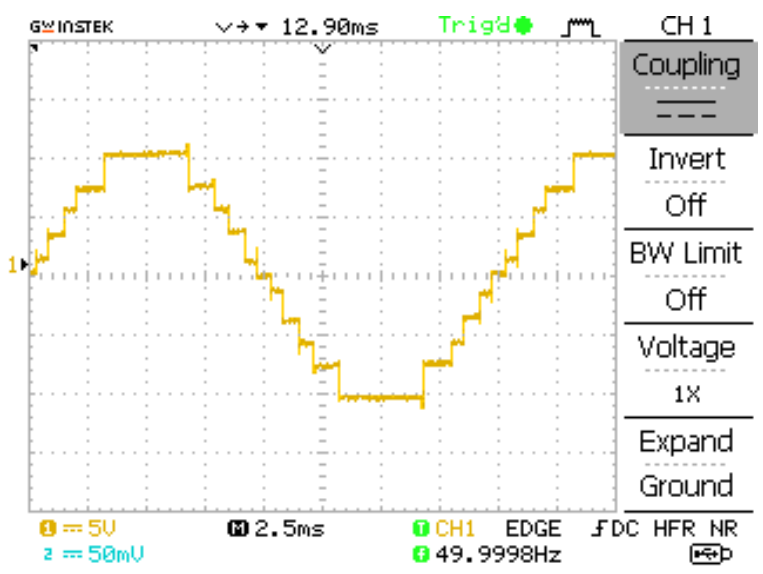

(a)

Fig 7: (a) Measured (b) Simulated phase voltage waveform for $V_{1}=1 P . U$

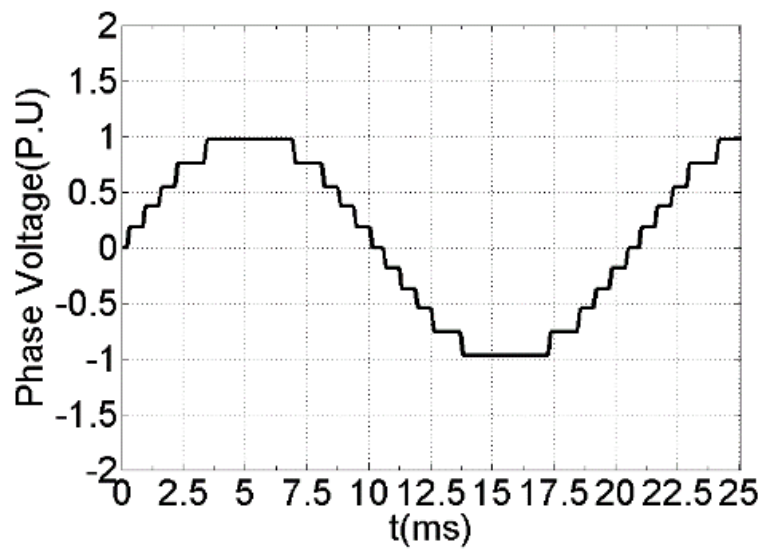

(b)

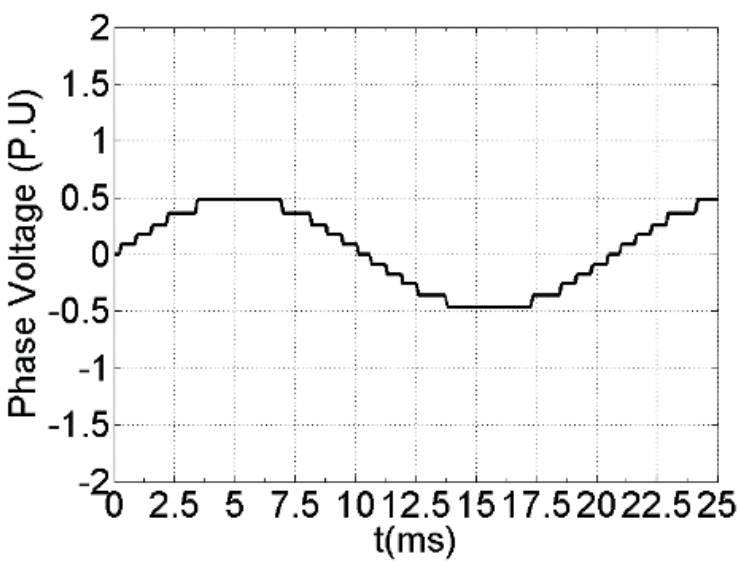

(b)

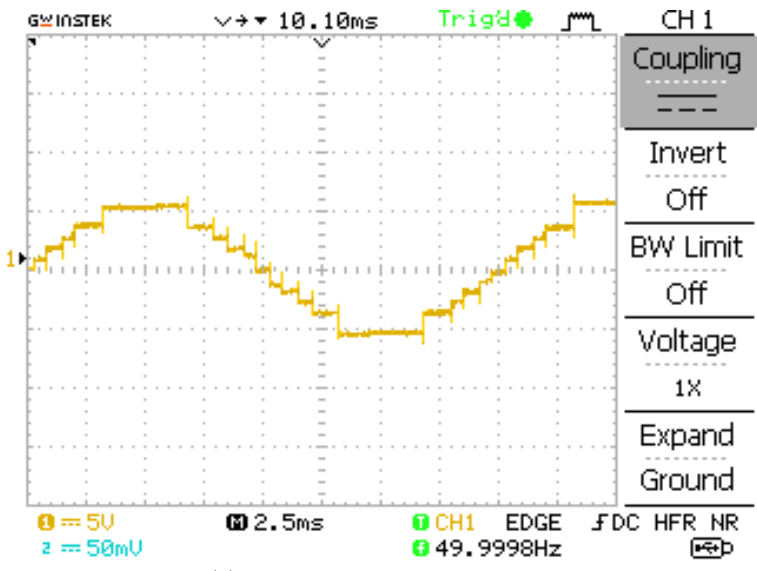

(a)

Fig 8: (a) Measured (b) Simulated phase voltage waveform for $\mathrm{V}_{1}=0.5 \mathrm{P} . \mathrm{U}$. 


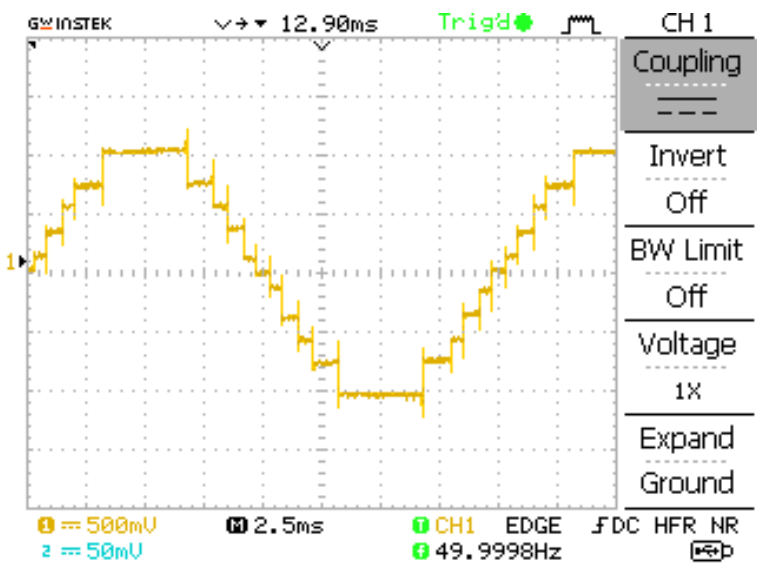

(a)

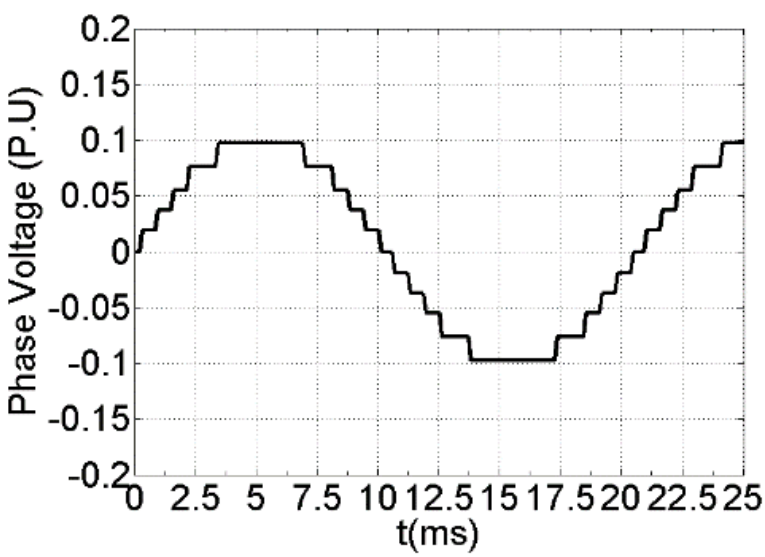

(b)

Fig 9 (a): Measured (b) Simulated phase voltage waveform for $V_{1}=0.1 P$.U.

\section{CONCLUSION}

This paper, proposes a new concept in switching of cascaded H-Bridge multilevel inverters. DC voltages of the inverter are considered to be dependent on switching angles. A polynomial term is considered as the relation between switching angles and DC voltages to independently control the switching pattern of the inverter regardless of its output voltage. Based on this concept, THD calculations are updated and proved to be independent from the fundamental voltage. Simulation results are verified through an experimental set up and the results show considerable extension in the range of output voltage while THD is kept at the minimum value.

\section{REFERENCES}

T. Poompavai and P. Vijayapriya, "Comparative analysis of modified multilevel DC link inverter with conventional cascaded multilevel inverter fed induction motor drive," in Energy Procedia, 2017.

[2] J. Jana, H. Saha, and K. Das Bhattacharya, "A review of inverter topologies for single-phase grid-connected photovoltaic systems," Renew. Sustain. Energy Rev., vol. 72, no. April 2015, pp. 12561270, 2017.

[3] R. Mahalakshmi and K. C. S. Thampatty, "Grid Connected Multilevel Inverter for Renewable Energy Applications," Procedia Technol., 2015.

[4] M. Quraan, T. Yeo, and P. Tricoli, "Design and Control of Modular Multilevel Converters for Battery Electric Vehicles," IEEE Trans. Power Electron., 2016.

[5] O. A. Gashteroodkhani, B. Vahidi, and A. Zaboli, "Time-time matrix z-score vector-based fault analysis method for seriescompensated transmission lines," Turkish Journal of Electrical Engineering \& Computer Sciences, vol. 25, pp. 2647-2659, 2017. S. M. R. Tousi and S. Aznavi, "Performance optimization of a STATCOM based on cascaded multi-level converter topology using multi-objective Genetic Algorithm," in Electrical Engineering (ICEE), 2015 23rd Iranian Conference on, 2015, pp. $1688-1693$.

O. A. Gashteroodkhani, M. Majidi, M. Etezadi-Amoli, A. F. Nematollahi, B. Vahidi, "A hybrid SVM-TT transform-based method for fault location in hybrid transmission lines with underground cables" Electric Power Systems Research, vol. 170, pp. 205-214, 2019.

[8] H. Zhang, A. Von Jouanne, S. Dai, A. K. Wallace, and F. Wang, "Multilevel inverter modulation schemes to eliminate commonmode voltages," IEEE Trans. Ind. Appl., 2000.

[9] J. Rodríguez, J. S. Lai, and F. Z. Peng, "Multilevel inverters: A survey of topologies, controls, and applications," IEEE Trans. Ind. Electron., 2002.

[10] S. Aznavi, P. Fajri, M. Benidris and B. Falahati, "Hierarchical droop controlled frequency optimization and energy management of a grid-connected microgrid," in 2017 IEEE Conference on Technologies for Sustainability (SusTech), Phoenix, AZ, USA 2017, pp. 1-7.
[11] A. Ruderman, "About voltage total harmonic distortion for singleand three-phase multilevel inverters," IEEE Trans. Ind. Electron., 2015

[12] A. M. Gole, "Sinusoidal Pulse width modulation," in Electronics, 2000.

[13] L. M. Tolbert and T. G. Habetier, "Novel multilevel inverter carrier-based PWM method," IEEE Trans. Ind. Appl., 1999.

[14] Z. Liu, Y. Wang, G. Tan, H. Li, and Y. Zhang, "A Novel SVPWM Algorithm for Five-Level Active Neutral-Point-Clamped Converter," IEEE Trans. Power Electron., 2016.

[15] K. Yang et al., "Unified Selective Harmonic Elimination for Multilevel Converters," IEEE Trans. Power Electron., 2017.

[16] M. Mirmozaffari and A. Alinezhad, "Ranking of heart hospitals using cross-efficiency and two-stage DEA," 2017 7th International Conference on Computer and Knowledge Engineering (ICCKE), Mashhad, 2017, pp. 217-222.

[17] M. Mirmozaffari, "Eco-Efficiency Evaluation in Two-Stage Network Structure: Case Study: Cement Companies". Iranian Journal of Optimization (IJO). Dec. 16, 2018.

[18] H. Firouzkouhi, " Application of Flexible OMTHD Technique to Cascaded Multi-level Inverter and the FPGA Based Implementation of Control System", International Research Journal of Engineering and Technology (IRJET), Vol. 05, Issue. 05, pp.1916-1922, May-2018. 